\title{
Chemo-dynamical simulations of galaxies
}

\author{
Chiaki Kobayashi \\ The Australian National University, Mt. Stromlo Observatory, Cotter Rd., Weston ACT \\ 2611, Australia, e-mail: chiaki@mso . anu. edu.au
}

\begin{abstract}
We predict the frequency distribution of elemental abundance ratios from Carbon to Zinc as a function of time and location, which can be directly compared with the next generation of instruments in the galactic archaeology project such as the HERMES. We perform the chemodynamical simulations of a Milky Way-type galaxy from a CDM initial condition, using a self-consistent hydrodynamical code with supernova feedback and chemical enrichment. In the simulated galaxy, the kinematical and chemical properties of the bulge, disk, and halo are consistent with the observations. The bulge stars have formed from the assembly of subgalaxies at $z \gtrsim 2$, and have higher $[\alpha / \mathrm{Fe}]$ ratios because of the lack of contribution of Type Ia Supernovae. The disk stars have formed at a constant rate of star formation over $13 \mathrm{Gyr}$, and show a decreasing trend of $[\alpha / \mathrm{Fe}]$ and increasing trends of $[(\mathrm{Na}, \mathrm{Al}, \mathrm{Cu}, \mathrm{Mn}) / \mathrm{Fe}]$. However, the thick disk stars tend to have higher $[\alpha / \mathrm{Fe}]$ and lower $[\mathrm{Mn} / \mathrm{Fe}]$ than thin disk stars. $60 \%$ of the thick disk stars formed in the satellite galaxies before they were accreted on to the disk in this CDM-based simulation.
\end{abstract}

Key words. Galaxy: abundances — Galaxy: evolution — stars: supernovae

\section{Introduction}

While the evolution of the dark matter is reasonably well understood, the evolution of the baryonic component is much less certain because of the complexity of the relevant physical processes, such as star formation and feedback. With the commonly employed schematic star formation criteria alone, the predicted star formation rates (SFRs) are higher than what is compatible with the observed luminosity density. Thus feedback mechanisms are in general invoked to reheat gas and suppress star formation. We include the feedback from stellar winds, core-collapse supernovae (normal Type II Supernovae (SNe II) and hypernovae $(\mathrm{HNe})$ ), and Type Ia Supernovae (SNe Ia) in

Send offprint requests to: Chiaki Kobayashi our hydrodynamical simulations. Supernovae inject not only thermal energy but also heavy elements into the interstellar medium (ISM), which can enhance star formation. Chemical enrichment must be solved as well as energy feedback. Supernova feedback is also important for solving the angular momentum problem and the missing satellite problem, and for explaining the existence of heavy elements in the intracluster medium and intergalactic medium, and possibly the mass-metallicity relation of galaxies (Kobayashi et al.2007).

In the next decade, high-resolution multiobject spectroscopy (HERMES) and space astrometry missions (GAIA) will provide the kinematics and chemical abundances of a million stars in the Local Group. Since different heavy elements are produced from different 


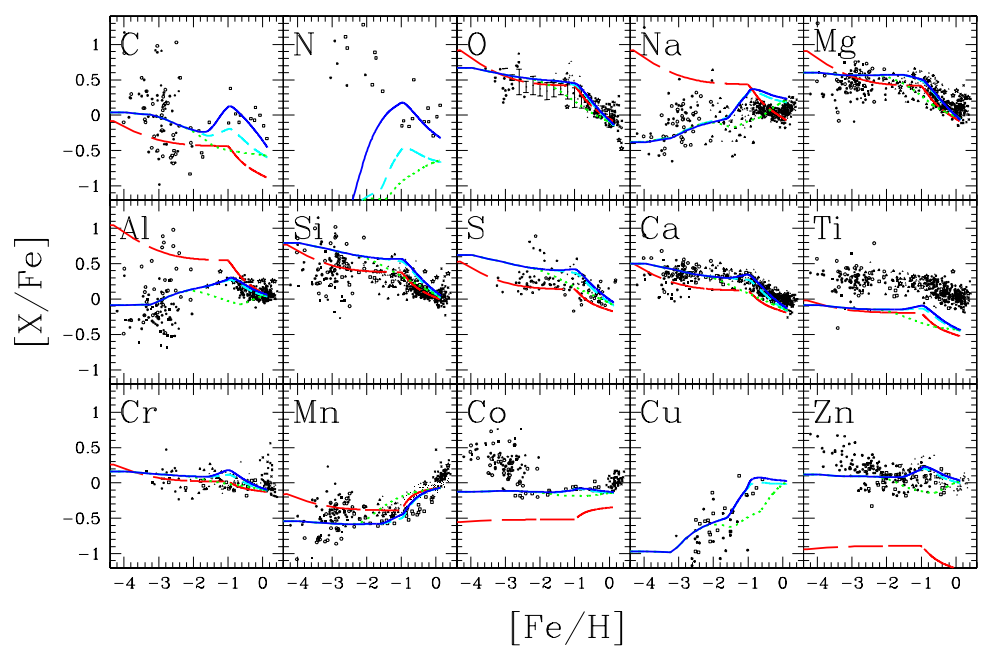

Fig. 1. The evolution of heavy element abundance ratios $[\mathrm{X} / \mathrm{Fe}]$ against $[\mathrm{Fe} / \mathrm{H}]$ for one-zone models with only SNe II (long-dashed line), with our new yields and SN Ia model (short-dashed line), with the double-degenerate scenario of SNe Ia (dotted line), and with AGB stars (solid line). The dots are observational data (see K06 for the references).

supernovae with different timescales, elemental abundance ratios can provide independent information on "age". Therefore, the stars in a galaxy are the fossils that can untangle the galactic history. The galactic archaeology technique can be used to study galaxy formation and evolution in general. Metallicities are measured in various objects with different galaxy mass scales and as a function of redshift/time. The internal structure of galaxies are being observed with integral field spectrographs (e.g., the SAURON project, SINFONI on VLT). In order to untangle the formation and evolution history of the galaxy from observational data, a "realistic" model that includes star formation and chemical enrichment is required.

\section{Chemical enrichment sources}

Hypernovae - The explosion mechanism of core-collapse supernovae is still uncertain, although a few groups have succeeded in exploding core-collapse supernovae. However, the ejected explosion energy and ${ }^{56} \mathrm{Ni}$ mass (which decays to ${ }^{56} \mathrm{Fe}$ ) can be directly estimated from the observations, i.e., the light curve and spectra fitting of individual supernova. As a result, it is found that many core-collapse supernovae $\left(M \geq 20 M_{\odot}\right)$ have more than ten times the ex- plosion energy $\left(E_{51}>10\right)$ and produce a significant amount of iron. We calculate the nucleosynthesis yields for wide ranges of metallicity $\left(Z=0-Z_{\odot}\right)$ and the explosion energy (normal $\mathrm{SNe}$ II and $\mathrm{HNe}$ ). Assuming that a large fraction of supernovae with $M \geq 20 M_{\odot}$ are $\mathrm{HNe}$, the evolution of the elemental abundance ratios from oxygen to zinc are in excellent agreement with observations in the solar neighborhood, bulge, halo, and thick disk (Kobayashi et al. 2006, hereafter K06).

Fig. 1 shows the evolution of heavy element abundance ratios $[\mathrm{X} / \mathrm{Fe}]$ against $[\mathrm{Fe} / \mathrm{H}]$ with our new yields (short-dashed lines), and with only SNe II (long-dashed lines, Nomoto et al. (1997)'s yields adopted). In the early stage of galaxy formation only SNe II explode, and $[\alpha / \mathrm{Fe}]$ stays constant. Because of the delayed Fe production by $\mathrm{SNe} \mathrm{Ia},[\alpha / \mathrm{Fe}]$ decreases toward 0. $\alpha$-elements, $\mathrm{O}, \mathrm{Mg}, \mathrm{Si}, \mathrm{S}$, and $\mathrm{Ca}$, show the plateau at $[\mathrm{Fe} / \mathrm{H}] \lesssim-1$. Ti is underabundant overall, which will be solved with the $2 \mathrm{D}$ calculation of nucleosynthesis. The observed decrease in the odd- $\mathrm{Z}$ elements $(\mathrm{Na}, \mathrm{Al}$, and $\mathrm{Cu})$ toward low $[\mathrm{Fe} / \mathrm{H}]$ is reproduced by the metallicity effect on nucleosynthesis. The ironpeak elements $(\mathrm{Cr}, \mathrm{Mn}, \mathrm{Co}$, and $\mathrm{Ni}$ ) are consis- 

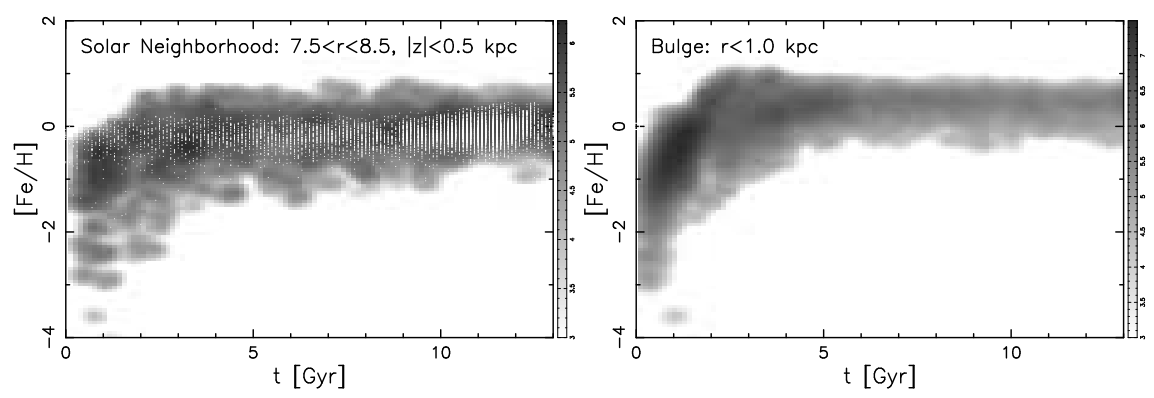

Fig. 2. Age-metallicity relations in the solar neighborhood (left panel) and bulge (right panel). The contours show the frequency distribution of stars in the simulated galaxy. The dots show the observations (Holmberg et al.2007).

tent with the observed mean values at $-2.5<$ $[\mathrm{Fe} / \mathrm{H}]_{\sim}<-1$, and the observed trend at the lower metallicity can be explained by the energy effect under the assumption of inhomogeneous enrichment. Note that $\mathrm{Cr}$ II data are plotted.

The most important improvement is in $\mathrm{Zn}$. The observed abundance of $\mathrm{Zn}([\mathrm{Zn} / \mathrm{Fe}] \sim$ $0)$ can be explained only by a large contribution of $\mathrm{HNe}$. Since the observed $[\mathrm{Zn} / \mathrm{Fe}]$ shows an increase toward lower metallicity, the $\mathrm{HNe}$ fraction may be larger in the earlier stage of galaxy formation. At high metallicity, since neutron-rich isotopes ${ }^{66-70} \mathrm{Zn}$ are produced, the $\mathrm{HNe}$ fraction can be as small as $1 \%$. In the following chemodynamical simulations, we adopt $\epsilon_{\mathrm{HN}}=0.5,0.5,0.4,0.01,0.01$ for $Z=$ $0,0.001,0.004,0.02,0.05$, which gives better agreement with the observed present hypernova rate. Pair-instability supernovae, which produce much more $\mathrm{Fe}$, more $[\mathrm{S} / \mathrm{Fe}]$, and less $[\mathrm{Zn} / \mathrm{Fe}]$, should not contribute in the galactic chemical evolution.

Type Ia Supernovae - The progenitors of the majority of $\mathrm{SNe}$ Ia are most likely the Chandrasekhar $(\mathrm{Ch})$ mass white dwarfs (WDs). For the evolution of accreting $\mathrm{C}+\mathrm{O}$ WDs toward the Ch mass, two scenarios have been proposed; one is the double-degenerate scenario, i.e., merging of double $\mathrm{C}+\mathrm{O}$ WDs with a combined mass surpassing the $\mathrm{Ch}$ mass limit. However, it has been theoretically suggested that it leads to accretion-induced collapse rather than SNe Ia. The other is our single-degenerate (SD) scenario, i.e., the WD mass grows by accretion of hydrogen-rich matter via mass transfer from a binary companion.

We construct a new model of $\mathrm{SNe}$ Ia, based on the SD scenario, taking account of the metallicity dependences of the WD wind (Kobayashi et al. 1998) and the massstripping effect on the binary companion star (Kobayashi \& Nomoto 2009). Our model naturally predicts that the $\mathrm{SN}$ Ia lifetime distribution spans a range of $0.1-20 \mathrm{Gyr}$ with the double peaks; the main-sequence+WD systems with the timescale of $\sim 0.1-1$ Gyr are dominant in star-forming galaxies, while the red-giants+WD systems with $\sim 1-20 \mathrm{Gyr}$ timescales are dominant in early-type galaxies.

From $[\mathrm{Fe} / \mathrm{H}] \sim-1, \mathrm{SNe}$ Ia start to occur producing more $\mathrm{Fe}$ than $\alpha$-elements, and thus $[\alpha / \mathrm{Fe}]$ decreases toward the solar abundance. The decreasing $[\mathrm{Fe} / \mathrm{H}]$ depends on the SN Ia progenitor model. Our SN Ia model can give better reproduction of the $[(\alpha, \mathrm{Mn}, \mathrm{Zn}) / \mathrm{Fe}]-$ $[\mathrm{Fe} / \mathrm{H}]$ relations in the solar neighborhood than other models such as the DD scenario (dotted lines). With the DD scenario, the typical lifetimes of $\mathrm{SNe}$ Ia are $\sim 0.1 \mathrm{Gyr}$, which results in the too early decrease in $[\alpha / \mathrm{Fe}]$ at $[\mathrm{Fe} / \mathrm{H}]$ $\sim-2$. Even with our SD model, if we do not include the metallicity effect, $[\alpha / \mathrm{Fe}]$ decreases too early because of the shortest lifetime, $\sim 0.1$ Gyr. In other words, the metallicity effect is more strongly required in the presence of the young population of $\mathrm{SNe}$ Ia.

For SNe Ia, we take the nucleosynthesis yields from Nomoto et al. (1997), where the metallicity dependence is not included. $\mathrm{Ni}$ is 

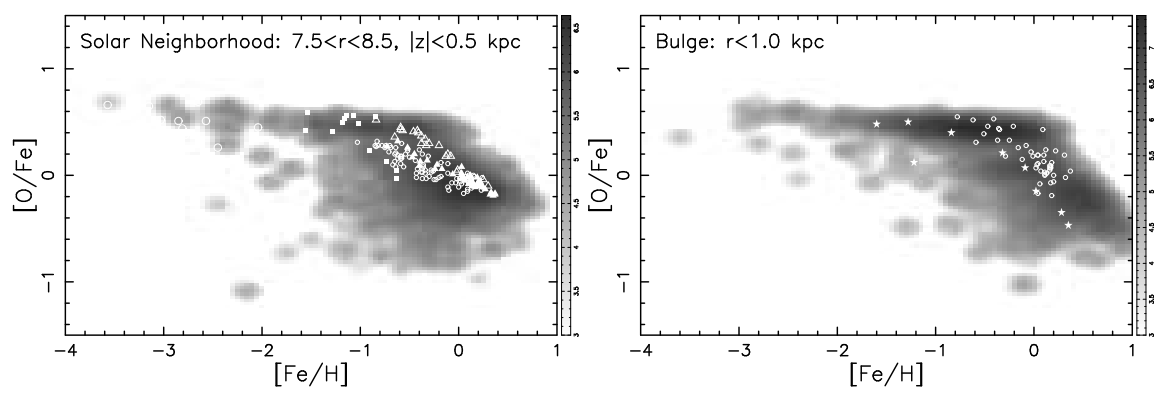

Fig. 3. $[\mathrm{O} / \mathrm{Fe}]-[\mathrm{Fe} / \mathrm{H}]$ relations in the solar neighborhood (left panel) and bulge (right panel). The contours show our simulation, and the dots are observational data (see KN10 for the references).

overproduced at $[\mathrm{Fe} / \mathrm{H}] \gtrsim-1$, which will be solved by tuning the propagation speed of the burning front and the central density of the white dwarf.

Asymptotic Giant Branch stars - Stars with initial masses between about $0.8-8 M_{\odot}$ (depending on metallicity) produce light elements such as $\mathrm{C}$ and $\mathrm{N}$, while the contribution of heavier elements are negligible in the galactic chemical evolution (solid lines). The nucleosynthesis yields of AGB stars involve uncertainties due to convection and mass loss. We introduce the new calculation by Karakas (2010). The Na overproduction problem has been solved with the updated reaction rates.

\section{The Milky Way Galaxy}

We simulate the chemodynamical evolution of the Milky Way-type galaxy from the CDM initial fluctuation (see Kobayashi \& Nakasato 2010, hereafter KN10, for the details). After the start of the simulation, the system expands according to the Hubble flow. The CDM initial fluctuations grow into the structures of nodes and filaments, and small collapsed halos are realized both in dark matter and gas. In the halos, the gas is allowed to cool radiatively, and star formation takes place since $z \sim 15$ According to the hierarchical clustering of dark halos, subgalaxies merge to form large galaxies, which induces the initial starburst. Under the CDM picture, any galaxy forms through the successive merging of subgalaxies with various masses. In this simulated galaxy, the bulge is formed by the initial starburst that is induced by the assembly of gas-rich sub-galaxies at $z \geq 3$. Because of the angular momentum, the gas accretes onto the plane forming a rotationally supported disk that grows from the inside out. In the disk, star formation takes place with a longer timescale, which is maintained not by the slow gas accretion, but by selfregulation due to supernova feedback. Many satellite galaxies come in successively and disrupt the disk, but there is no major merger event after $z \sim 2$, which is necessary in order to retain the disk structure. Metallicity gradients, increasing toward higher density regions, are generated both in the gas phase and stars from $z \sim 5$ onwards.

The bulge has a de Vaucouleurs surface brightness profile with an effective radius of $\sim 1.5 \mathrm{kpc}$, and the disk has an exponential profile with a scale length of $\sim 5 \mathrm{kpc}$. In the following, we define the three major components simply from the location of the stars at the present-day: the radius of $7.5 \leq r \leq 8.5$ $\mathrm{kpc}$ and the height of $|z| \leq 0.5 \mathrm{kpc}$ for the solar neighborhood, $r \leq 1 \mathrm{kpc}$ for the bulge, and $5 \leq r \leq 10 \mathrm{kpc}$ for the halo. The thick disk stars are defined from the kinematics: the ratio between rotation velocity and velocity dispersion $v / \sigma<1$ in the solar neighborhood.

The resultant star formation histories are different for different components. In the bulge, most of stars have formed in the first 2 Gyr. $80 \%$ of stars are older than $10 \mathrm{Gyr}$, and $60 \%$ have $[\mathrm{O} / \mathrm{Fe}]>0.3$. In the disk, $50 \%$ of solar-neighborhood stars are younger than $8 \mathrm{Gyr}$, and $80 \%$ have $[\mathrm{O} / \mathrm{Fe}]<0.3$. The thick disk stars tend to be older and have higher $[\alpha / \mathrm{Fe}]$ than the thin disk stars. The formation timescale of the thick disk is 4 Gyr. 

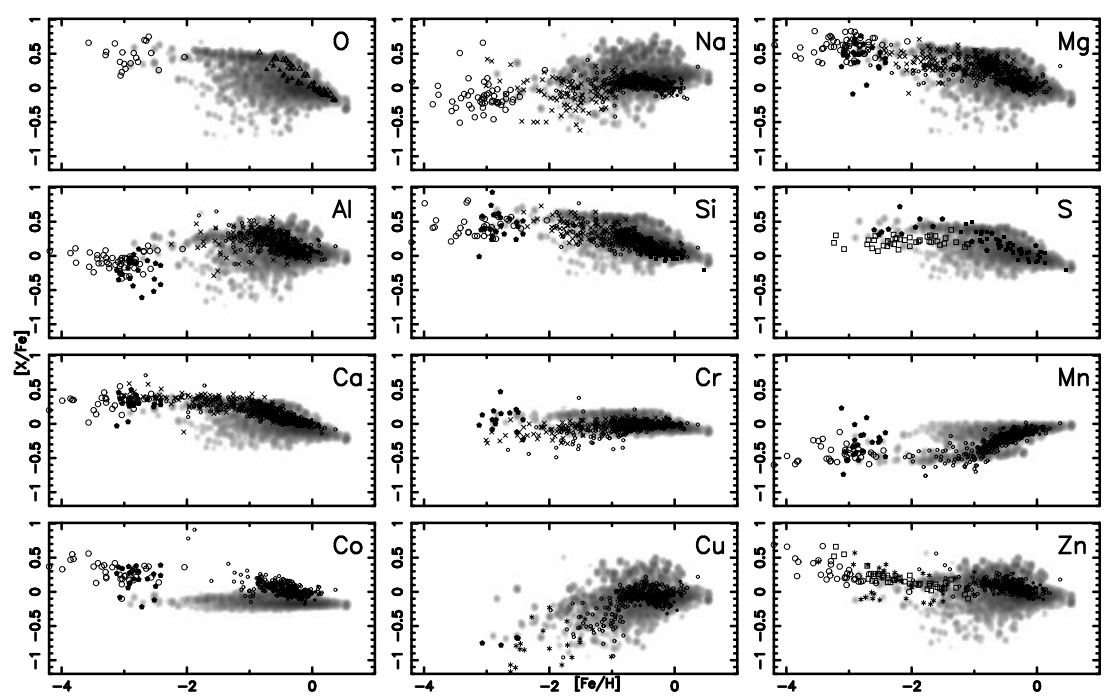

Fig. 4. $[\mathrm{X} / \mathrm{Fe}]-[\mathrm{Fe} / \mathrm{H}]$ relations in the solar neighborhood.

\subsection{Age-metallicity relations}

The chemical enrichment timescale is also different for the different components. Fig. 2 shows the age-metallicity relations. In the solar neighborhood, the average metallicity reaches $[\mathrm{Fe} / \mathrm{H}] \sim 0$ at $t \sim 2 \mathrm{Gyr}$, and does not show strong evolution for $t \gtrsim 2$ Gyr. The scatter in metallicity at a given time is caused by the inhomogeneity of chemical enrichment in our chemodynamical model; there is a local variation in star formation, metal production by supernovae, and metal flow by the inflow and outflow of the ISM. As a result, both the average and scatter are in excellent agreement with the observations (dots) in spite of the uncertainties in the observational estimates of the ages.

In the bulge, star formation takes place more quickly, and thus the chemical enrichment timescale is much shorter than in the disk. The age-metallicity relation shows a more rapid increase than in the disk. The maximum metallicity reaches super solar $([\mathrm{Fe} / \mathrm{H}] \sim 1)$ at $t \sim 2$ Gyr. Although the SFR becomes small after $\sim 5 \mathrm{Gyr}$, a few stars form at $>5 \mathrm{Gyr}$. These have super-solar metallicity in general and the average metallicity do not show time evolution.

\section{2. $[\alpha / \mathrm{Fe}]-[\mathrm{Fe} / \mathrm{H}]$ relations}

The difference in the chemical enrichment timescales results in a difference in the ele- mental abundance ratios. The best known clock is the $\alpha$-elements to iron ratio $([\alpha / \mathrm{Fe}])$ since SNe Ia produce more iron than $\alpha$ elements with longer timescales than SNe II. Nevertheless it should be noted that low-mass SNe II (10 $\left.13 M_{\odot}\right)$ also provide relatively low $[\alpha / \mathrm{Fe}]$ because of their smaller envelope mass compared to more massive SNe II. This mass dependence is also important in dwarf spheroidal galaxies.

Fig. 3 shows the $[\mathrm{O} / \mathrm{Fe}]-[\mathrm{Fe} / \mathrm{H}]$ relations, and the other $\alpha$-elements show the same trends. In the solar neighborhood, the evolutionary trend is in great agreement with the observations (dots). A significant scatter is seen, which is caused by the inhomogeneity of chemical enrichment in our chemodynamical model. At $[\mathrm{Fe} / \mathrm{H}] \geq-1$, the majority of $[\mathrm{O} / \mathrm{Fe}]$ is lower than 0.2 and the peak $[\mathrm{O} / \mathrm{Fe}]$ is -0.15 . Around $[\mathrm{Fe} / \mathrm{H}] \sim-1$, the scatter looks a bit larger than observed (Fig. 3). This may be because the mixing of heavy elements among gas particles is not included in our model. At $[\mathrm{Fe} / \mathrm{H}]<-1$, the scatter is caused from $\mathrm{SNe}$ Ia and/or low mass $\mathrm{SNe}$ II in the inhomogeneous enrichment in our chemodynamical models. The $[\alpha / \mathrm{Fe}]$ scatter in the simulation could be larger, since there is also a variation depending on the explosion energy and the remnant mass (neutron star and blackhole) for $\mathrm{SNe}$ II and $\mathrm{HNe}$ (faint $\mathrm{SNe}$ ). In other words, statistical comparison 


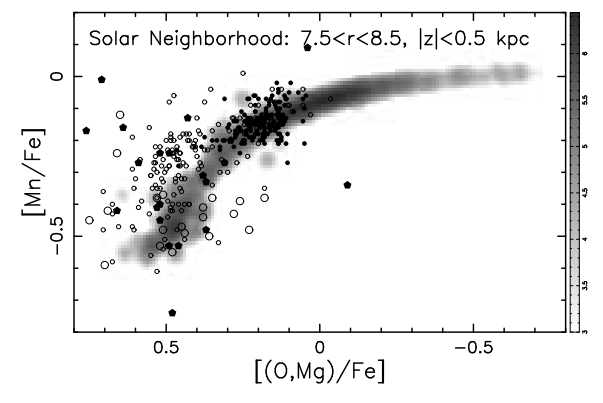

Fig. 5. The $[\alpha / \mathrm{Fe}]-[\mathrm{Mn} / \mathrm{Fe}]$ relation.

with the observed scatter could provide constraints on the unsolved physics of supernova explosions.

In the bulge, the $[\alpha / \mathrm{Fe}]-[\mathrm{Fe} / \mathrm{H}]$ relation is very much different. The chemical enrichment timescale is so short that the metallicity reaches super solar before $\mathrm{SNe}$ Ia can contribute. Thus, the $[\alpha / \mathrm{Fe}]$ plateau continues to $[\mathrm{Fe} / \mathrm{H}] \sim+0.3$. This is roughly consistent with the observations. In this simulated galaxy, some new stars are still forming in the bulge, which in general have super solar metallicity and low $[\alpha / \mathrm{Fe}]$ because of the large contribution from SNe Ia. Although a small fraction of stars with the age of $\sim 3$ Gyr $(t \sim 10.3 \mathrm{Gyr})$ do have high $[\alpha / \mathrm{Fe}]([\mathrm{O} / \mathrm{Fe}]=0.3)$, which is caused by the inhomogeneous enrichment, stars younger than $1 \mathrm{Gyr}$ have $-0.5 \leq[\mathrm{O} / \mathrm{Fe}]$ $\leq 0$ and $0 \leq[\mathrm{Fe} / \mathrm{H}] \leq 0.8$.

\section{3. $[\mathrm{X} / \mathrm{Fe}]-[\mathrm{Fe} / \mathrm{H}]$ diagrams}

We can predict the frequency distributions of the elements from $\mathrm{O}$ to $\mathrm{Zn}$ as a function of time and location. Fig. 4 shows the mass density of stars in the $[\mathrm{X} / \mathrm{Fe}]-[\mathrm{Fe} / \mathrm{H}]$ diagrams for the solar neighborhood (see KN10 for the bulge and thick disk) at present. Because of the delayed enrichment of SNe Ia, $\alpha$ elements $(\mathrm{O}, \mathrm{Mg}, \mathrm{Si}$, $\mathrm{S}$, and $\mathrm{Ca})$ show a plateau at $[\mathrm{Fe} / \mathrm{H}] \sim-1$, and then the decreasing trend against $[\mathrm{Fe} / \mathrm{H}]$, where $[\mathrm{Mn} / \mathrm{Fe}]$ also shows the increasing trend. Odd- $\mathrm{Z}$ elements ( $\mathrm{Na}, \mathrm{Al}$, and $\mathrm{Cu}$ ) show the increasing trend at $[\mathrm{Fe} / \mathrm{H}]<-1$ because of the metallicity dependence of nucleosynthesis yields. These are in excellent agreement with the available observations. In the bulge, the star formation timescale is so short that the $[\alpha / \mathrm{Fe}]$ plateau continues to $[\mathrm{Fe} / \mathrm{H}] \sim+0.3$. Because of the smaller contribution from $\mathrm{SNe} \mathrm{Ia}$, the majority of stars show high $[\alpha / \mathrm{Fe}]$ and low $[\mathrm{Mn} / \mathrm{Fe}] .[(\mathrm{Na}, \mathrm{Al}, \mathrm{Cu}, \mathrm{Zn}) / \mathrm{Fe}]$ are also high because of the high metallicity in the bulge.

The stellar population of the thick disk is neither disk-like nor bulge-like. In the thick disk, $[\alpha / \mathrm{Fe}]$ is as high, and $[\mathrm{Mn} / \mathrm{Fe}]$ is as low, as in the bulge because of the short formation timescale. However, [( $\mathrm{Na}, \mathrm{Al}, \mathrm{Cu}, \mathrm{Zn}) / \mathrm{Fe}]$ are not as high as in the bulge because of the lower chemical enrichment efficiency. This is because half of the thick disk stars have already formed in satellite galaxies before they accrete onto the disk, and the metals have been ejected from the satellite galaxies by the galactic winds.

\section{4. $[\alpha / \mathrm{Fe}]-[\mathrm{Mn} / \mathrm{Fe}]$ diagram}

In Fig. 5 for the solar neighborhood, $[\mathrm{Mn} / \mathrm{Fe}]$ is plotted against $[\alpha / \mathrm{Fe}]=([\mathrm{O} / \mathrm{Fe}]+$ $[\mathrm{Mg} / \mathrm{Fe}]) / 2$, which clearly shows the sequence of SN Ia contribution. With $\mathrm{SNe}$ II and $\mathrm{HNe}$ only, $[\alpha / \mathrm{Fe}]$ is as high as $\sim 0.5$, and $[\mathrm{Mn} / \mathrm{Fe}]$ is as low as $\sim-0.5$. With more $\mathrm{SNe} \mathrm{Ia},[\alpha / \mathrm{Fe}]$ decreases, while $[\mathrm{Mn} / \mathrm{Fe}]$ increases. The three populations of observed stars follow this trend: i) the EMP stars (large open circles and filled pentagons) are found in the left-bottom region with high $[\alpha / \mathrm{Fe}]$ and low $[\mathrm{Mn} / \mathrm{Fe}]$;

ii) The thick disk stars (small open circles) populate the following region, $[\alpha / \mathrm{Fe}] \sim 0.2-0.4$ and $[\mathrm{Mn} / \mathrm{Fe}] \sim-0.4$ to -0.2 ;

iii) The thin disks stars (small closed circles) occur at $[\alpha / \mathrm{Fe}] \sim 0.1$ and $[\mathrm{Mn} / \mathrm{Fe}] \sim-0.1$, forming from the ISM largely enriched by SNe Ia.

In other words, it is possible to select thick disk stars only from the elemental abundance ratios.

\section{References}

Holmberg, J., et al. 2007, A\&A, 475, 519

Karakas, A. 2010, MNRAS, submitted

Kobayashi, C. \& Nomoto, K. 2009, ApJ, 707, 1466

Kobayashi, C. \& Nakasato, N. 2010, ApJ, submitted (KN10)

Kobayashi, C., Springel, V, \& White, S. D. M. 2007, MNRAS, 376, 1465

Kobayashi, C., et al. 2006, ApJ, 653, 1145

Kobayashi, C., et al. 1998, ApJ, 503, L155 